\title{
Sieving Extremely Wet Earth Mass by Means of Oscillatory Transporting Platform
}

\author{
Goran Mihajlović ${ }^{\mathrm{a}^{*}}$, Milutin Živković ${ }^{\mathrm{a}}$ \\ ${ }^{a}$ Higher Technical School of Mechanical Engineering, Radoja Krstića 19, 37240 Trstenik, Serbia
}

\begin{abstract}
The experience acquired from the exploitation has shown that problems in the work of oscillatory platforms appear after heavy rains, i.e. when the soil moisture $(w)$ is greatly increased. Then the effect of transportation and sieving is the weakest, due to sticking moist earth for grille of the platform. Therefore, the main aim of this article was to create a complex theoretical and practical mathematical model of the processes mentioned above, in order to test how the platform works in extreme working conditions after abundant and prolonged rains. Therefore, complete research is divided into two characteristic phases: theoretical phase and experimental phase. In the theoretical research phase, dynamic modeling of the process of sieving of extremely wet earth mass was performed using the transmissional function analysis method and the method of Laplace transforms [1]. The Novelty in the theoretical model given by Equation 3 is that the existing model for sieving dry earth mass [2] has been improved by introducing delay coefficient $K_{\tau}=[0 \ldots 1]$. In the experimental research phase, the original mathematical model was created using the multi-factor experiment plan method which will be shown, while the analysis of the experimental parameters was performed in three characteristic steps: regression analysis- dispersion analysis- decoding mathematical model [3].
\end{abstract}

Keywords:

Oscillatory Platform; Wet Earth Mass; Sieving; Mathematical Model; Stability;

Manageableness.

\section{1- Introduction}

Separation (segmentation) is a physical or chemical process in which some less important components (called "tailings") are removed, while the material itself is enriched with more valuable ingredients in order to be efficiently used both in technical and economical terms. Separation process individual component ingredients always precede the processes of breaking and grinding of solid mass [4] which are usually performed on machines called crusher (coarse and medium shredding) and mills (for fine shredding). At the same degree of fragmentation is the relationship between grain size after crushing and its size before crushing.

However, recently it was discovered that the process of breaking up and separating solid mass, besides the already mentioned methods, can also be performed on a very successful and rational manner by using special vibrating oscillatory transportation platform [5,6]. Since the beginning of the sixties the last century, great number of authors in the world dealt with the specific problems of increasing the working abilities of platforms, mainly intended for finegrained and dry materials. Their physical models had their developmental-dialectical trend starting from the simplest with one or two masses interconnected by elastic connections, to the most complex models which have been subjected to optimization of the most influential operating parameters. However, all mentioned models relate mainly to the transport of dry, mostly earthen mass and the same were a good starting point for creation the experimental model of the process of sieving of wet earth mass by means of oscillatory transporting platform, which is created by the authors of this article according to the methodology shown in Figure 3.

\footnotetext{
*CONTACT: Goran.vtms@gmail.com

DOI: http://dx.doi.org/10.28991/esj-2020-01221
}

(C) 2020 by the authors. Licensee ESJ, Italy. This is an open access article under the terms and conditions of the Creative Commons Attribution (CC-BY) license (https://creativecommons.org/licenses/by/4.0/). 
Stylized scheme of oscillatory transporting platform with characteristic geometric angles is shown in Figure 1, stylized schematic shew of the wet earth mass separation process is given in Figure 2, while methodology [7-11] for realization the experiments by the authors in order to obtain an adequate experimental model [3] is shown in Figure 3.

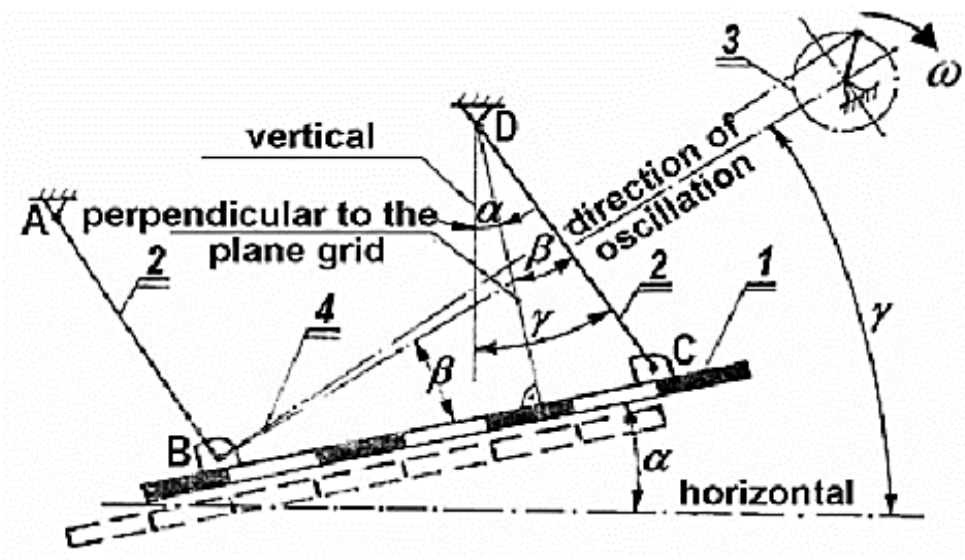

Figure 1. Stylized scheme of oscillatory transporting platform (1-platform; 2-suspension; 3-excenter; 4-driving lever; $A$ amplitude of oscillation of the platform (radius of the excenter); $\varphi$-phase of platform oscillation; $\alpha, \beta, \gamma$-influential angles of the platform; A, B, C, D-supporting points of the platform).

a

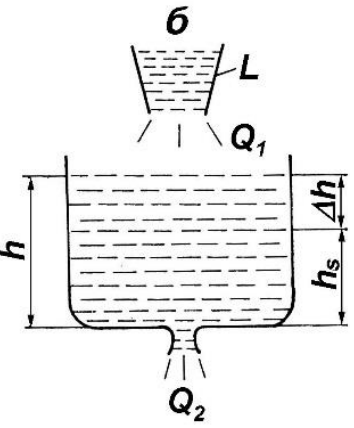

Figure 2. Schematic shew of the process of separation of wet earth mass by using oscillatory transporting platform.

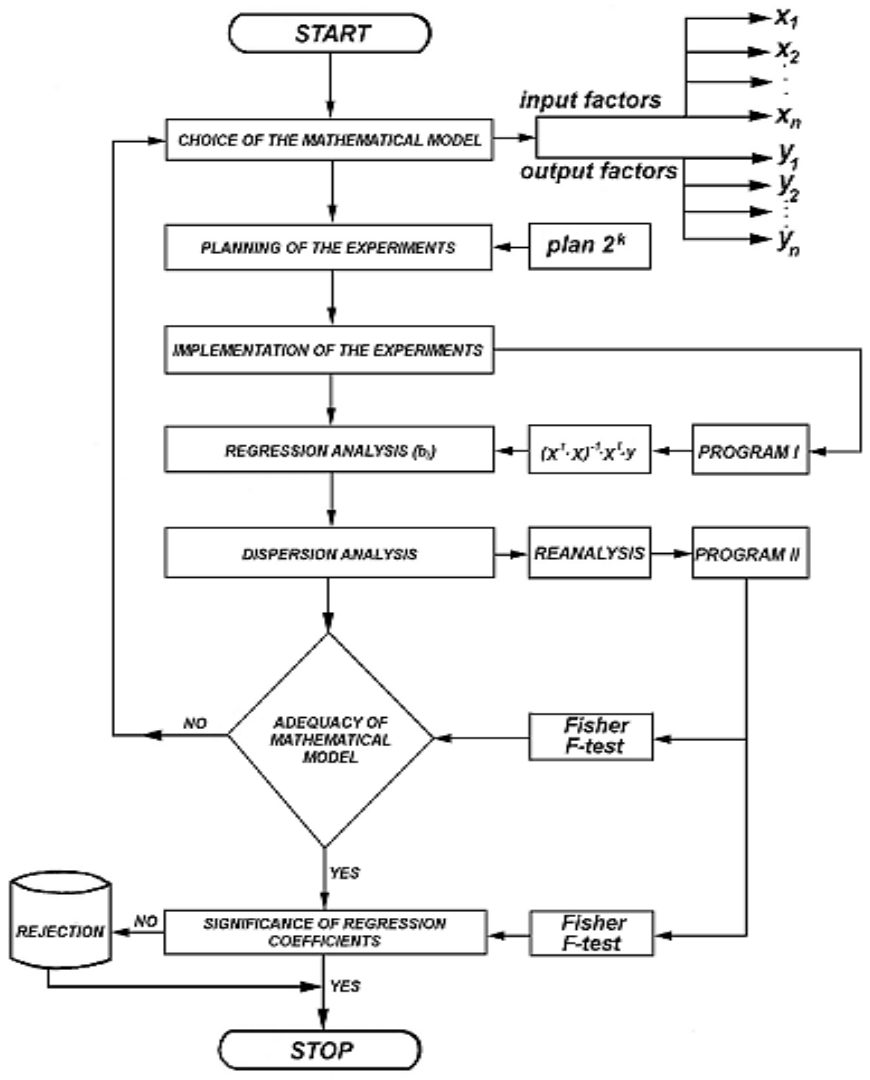

Figure 3. Methodology of realization of experiments. 
It is normal that the operation of the platform is influenced by many different factors, and considering that this is a relatively new and unknown issue, it is necessary to carry out extensive theoretical and practical research in order to obtain relevant results necessary for a complete understanding of the process mentioned above. The aim, as always in similar situations, the data obtained and conclusions drawn to be further take advantage for the design, construction and operation of real mechanical and technological systems, in which as well as the working elements are built-in the same or similar type of oscillatory platform.

In order to be able to analyze the operation of the oscillatory transporting platform, it is necessary to know the following parameters [13]:

$Q_{1}\left(m^{3} / s\right)$ - amount of delivered wet earth mass on the platform (power platform);

$Q_{2}\left(\mathrm{~m}^{3} / \mathrm{s}\right)$ - amount of sieved wet earth mass through the grid of the platform;

$P\left(\mathrm{~m}^{2}\right)$ - the total area of the grid platform through which is performed the wet sieving land mass, where is:

$P=P_{1}+P_{2}+\ldots+P_{n}=\sum_{i=1}^{n} P_{i}=\sum_{i=1}^{n}(l \cdot d)=\prod_{i=1}^{n}\left(n \cdot P_{i}\right)$

$P_{i}\left(m^{2}\right)$ - elementary surface of a (i-th) field of grid of platform through which is carried out the sieving of wet earth mass (Figure 2a);

$l(m)$ - longitude of grid platform through which is carried out the sieving of wet earth mass (Figure 2a);

$d(m)$ - size of the hole (perforation) on the grid of the platform (Figure 2a);

$n$ - the total number of elementary fields on a grid of the platform (Figure $2 a$ ).

If on the grate of the platform arrives dry and friable earth masses (the optimal conditions for the transport and separation of a solid mass), then the effect of separation and sieving is the maximal and the fineness coefficient of separation is close to one $\left(\eta \approx 1\right.$, i.e. $\left.Q_{1} \approx Q_{2}\right)$. Mass sieving begins practically at the same moment when the solid mass delivered the grid of the platform (no "delay" time).

However, if on the grid of the platform arrives wet and plastic earth mass (the most unfavorable conditions for transport and separation of solid mass), the fineness coefficient of separation rapidly decreases and in extreme cases tends to zero $(\eta \rightarrow 0)$, which can cause a complete loss of a working effect of platform machines. Then we can define a new parameter that expresses the amount of non-sieved wet earth mass $Q^{*}\left(\mathrm{~m}^{3} / \mathrm{s}\right)$, which is:

$Q^{*}=Q_{1}-Q_{2}$

Here is particularly important to note that the sieving of wet land mass will not start immediately when it is received on the grid of the platform, but after a certain time $t$, which is called a delay time of the output parameter $\left(Q_{2}\right)$ relative to the input parameter $\left(Q_{l}\right)$.

The reason for this delay is the sticking of wet land mass on grid of the platform, due to the strong cohesion and adhesion forces that contribute to the country between the particles and the lattice, creates a specific physico-chemical compound which is difficult to disassemble. As a measure of stickiness is used time delay $\tau$, that in mathematical model conditionally can be understood as value which would be analogous to a kind of "stickiness coefficient" which could be expressed the intensity of said stickiness. Then there is a basic rule that the intensity of stickiness higher if the delay time longer.

In Mihajlović (2014) [1] study the mathematical model of the process of separating the wet earth mass in function of time by means of oscillatory transporting platform was presented and given by the differential Equation 3 .

$T_{V} \frac{d y(t)}{d t}+\left(1-K_{\tau}\right) y(t)=K_{P} \cdot x(t-\tau)$

The individual sizes in Equation 3 have the following meanings:

$T_{V}$ - temporal (inertial) constant of the system E-P-WP (environment-platform-working parameters);

$K_{\tau}=[0 \ldots 1]-$ delay coefficient of the sieving earth mass relative to earth mass which is arrived on the platform;

$K_{P}-$ coefficient (factor) amplification of the system E-P-WP;

$\tau$ - time delay of the output-response signal of the system E-P-WP with respect to the input-excitation signal. 


\section{2- Necessary Conditions for Stability of the Oscillatory Transporting Platform during the Sieving Wet Earth Mass}

Due to the effects of different disturbance factors in the environment (disturbance forces and moments, the unfavorable composition of the soil, high humidity, etc.) resulting difference between the input size, i.e. amount of delivered wet earth mass on the platform $\left(Q_{1}\right)$ and output size, i.e. the actual amount of sieved wet earth mass through the grid of the platform $\left(Q_{2}\right)$. The resulting difference is necessary to adjust in some way.

It is particularly important to point out the fact that response time of the platform $t_{o p}$ which characterizes the speed of calming disturbed motion (duration of the transient process) does not depend on the type of disorder, but only from the design parameters of the platform. Depending on the duration of the easing "calming" platform upon any intentionally or accidentally induced disorders, in practice, there are two typical cases:

- I case: if it is $t_{o p}>t_{r p} \rightarrow$ correction by the open system;

- II case: if it is $t_{o p}<t_{r p} \rightarrow$ correction by the closed-loop feedback;

$t_{r p}$ - reaction time of platform (minimum time required to change the mode platform, by changing one or more operating parameters).

In the first case, if the response time platform $t_{o p}$ is very lengthy (slowly calming the after the disturbance) and if the too long since the reaction platform $t_{r p}$, platform cannot receive the signal output size, but it will perform control operations on a predetermined program. This would be a correction by the open system, where a correction may itself represent a new disorder, which is screening moist soil even more difficult (Figure 4).

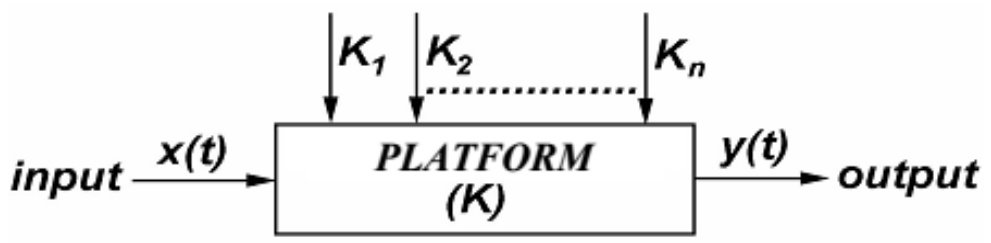

Figure 4. Structure scheme of an open system $E-P$ - WP (environment-platform-working parameters).

Tags in Figure 4 have the following meanings:

$x(t)$ - input values, which replaces the effect of environment on the observed system $E$ - $P$-WP (independent variable sizes);

$y(t)$ - output value, which replaces the effect of the system on the environment (depending on variable size);

$K$ - transformation operator, which are contained in all the elementary operations $\left(K_{1}, K_{2}, \ldots, K_{n}\right)$ which performed upon the input size $x(t)$ to obtain the output value $y(t)$.

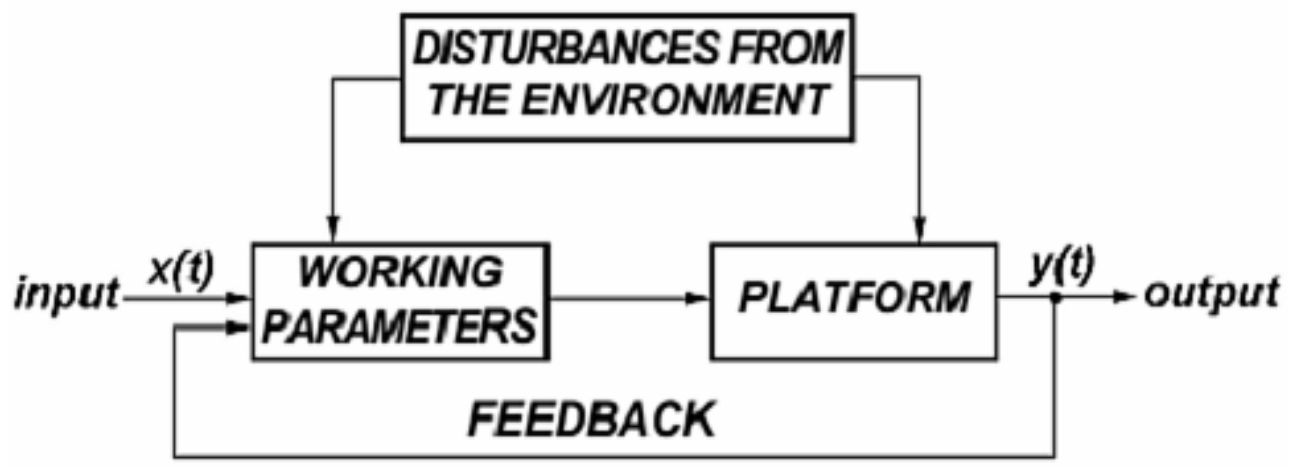

Figure 5. Structure scheme of a closed system $E-P$-WP (environment-platform-working parameters).

In another case, if the response time of the platform $t_{o p}$ is relatively short (quickly calming the after the disturbance) and if the same shorter than the platform reaction time $t_{r p}$, then, through a feedback signal can be transmitted output file to management system of the platform platform, to regime change its work sifting moist earth again closer to the desired (predetermined) value. This would be a typical correction by closed-loop feedback (Figure 5).

Stated above we can conclude the following [3]:

- In all types of disability (either from the environment or due to the reaction of the platform) is necessary to work the platform as quickly as possible calms and tends to a steady operating mode; 
- It is preferred that the response time of the platform and also the reaction time of the platform to be as low as possible (the shortest length), in order to achieve greater safety and reliability in operation, i.e. that the platform can in real time monitored all new circumstances;

- It is necessary, after calming down and after the completion of transition process, the platform be at operating mode that is as close as possible the desired operating mode sifting wet land mass.

Only after the fulfillment of all three of these requirements can be said to be the work of oscillatory transport platform develop as expected, and that the choice of operating parameters such that it creates a stable work platform from the aspect of separation and sieving of wet land mass.

\section{3- Response, Transmissional Function and "PN-diagram" of Oscillatory Transporting Platform during the Sieving of Wet Earth Mass}

The general solution in the function of the time of the differential Equation 3 is obtained as the sum of the corresponding homogeneous and particular solution and the same is the response of the oscillatory transport platform to deliberately or inadvertently caused disorder:

$$
y(t)=A e^{\left(\frac{K_{\tau}-1}{T_{V}}\right) t}+\frac{K_{P} \cdot x_{0}(t-\tau)}{1-K_{\tau}}
$$

The graphical interpretation of Equation 4 is given in Figure 6. From the figure it is noticed that output signal $y(t)$ starts to be generated only after a certain time $\tau$, which represents the time of its delay in relation to the input signal. Therefore, for the studied E-P-WP system, it can be said that it behaves as a typical aperiodic element first-order with delay, whose characteristics and dynamic behavior are well-known from the theory of linear systems [13].

It is believed that the transition process is completed when the function of the transitional characteristic $h(t)$ reaches value $h(t)=y(t) \approx 0.95 \cdot K_{P} \cdot x_{0}$ (which is approximately $t \approx 3 \cdot T_{V}$ ), because then establishes a new equilibrium of the system E-P-WP [14].
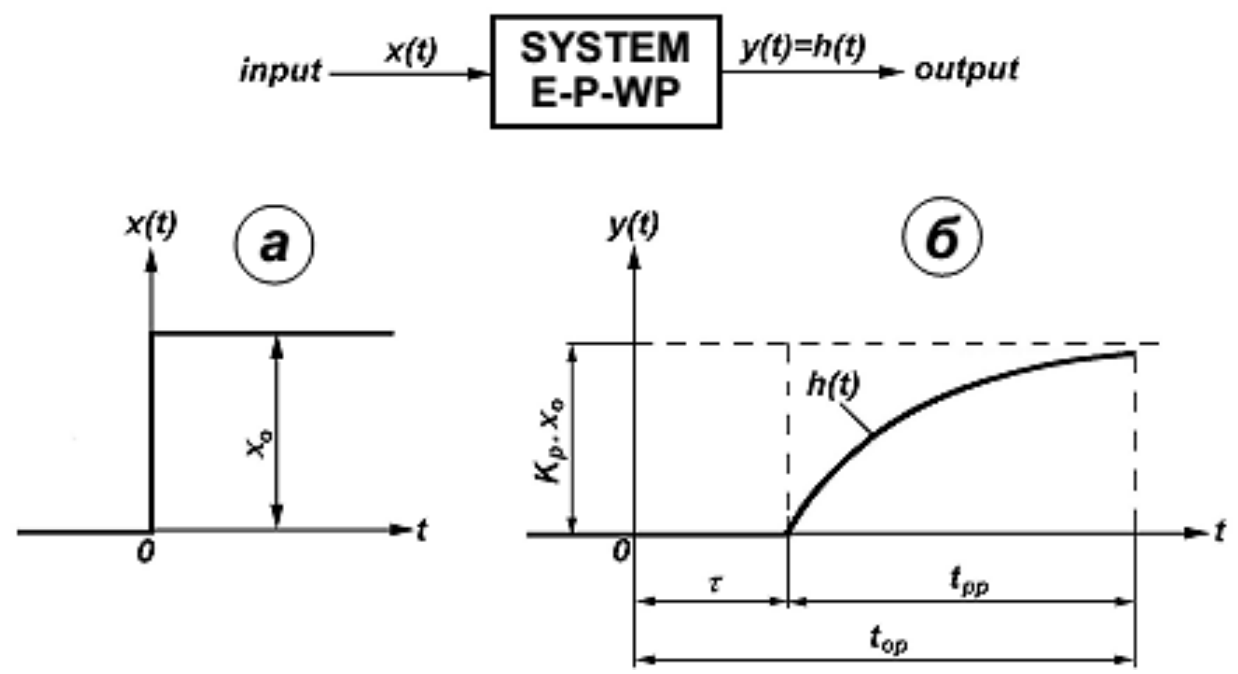

Figure 6. The response of oscillatory transporting platform during the sieving of wet earth mass due to disorder that is caused by jumping causal function ( $\tau$ - the delay time of the output signal relative to the input signal; $t_{p p}$ - length of the transition process; $t_{o p}$ - response time of the platform).

Transmissional function of the system E-P-WP get such a whatever perform Laplace transformation the left and right sides of the Equation 3 which is a mathematical model of the studied system:

$L\left[T_{V} \frac{d y(t)}{d t}+\left(1-K_{\tau}\right) y(t)\right]=L\left[K_{P} \cdot x(t-\tau)\right]$

whence arises:

$G(p)=\frac{K_{P}}{T_{V} \cdot p+1-K_{\tau}} e^{-\tau p}$

$e^{-\tau p}-$ a delay operator the output signal relative to the input signal (due to the sticking of wet earth mass for grid platforms). 
When the numerator in Equation 5 is equal to zero, it is obtained:

$G(p)=0$, for $K_{P} \cdot e^{-\tau p}=0 \Leftrightarrow K_{P}=0 \vee e^{-\tau p}=0$

In this case, the points $z_{i}(i=1,2, \ldots, n)$ in which transmissional function $G(p)$ takes the value of zero represent zero of transmissional function. However, because they are both amplification coefficient of the system $K_{P}$ and operator of delay $e^{-\tau p}$ always different from zero, comes to the conclusion that can never be filled condition $G(p)=0$, which means that transmissional function of the system E-P-WP no zero in the plane of complex variable $p=c+j \omega$.

When the denominator in Equation 5 is equal to zero and solve by " $p$ ", it is obtained:

$T_{V} \cdot p+1-K_{\tau}=0$

$p=\frac{K_{\tau}-1}{T_{V}}$

In this case, is valid already rendered statement that the case $K_{\tau}=1, p=0$, is mathematically possible but not physically, and it will not be considered for reason already explained [3].

Operator of delay that exists in Equation 5 in the case of the analyzed system E-P-WP has the following values:

$e^{-\tau p}=e^{-\tau\left(\frac{K_{\tau}-1}{T_{V}}\right)}=e^{\left(\frac{1-K_{\tau}}{T_{V}}\right) \tau}$

Zeros and poles of the transmissional function $G(p)$ can be displayed graphically on the same chart (Figure 7) in the plane of the complex variable $p=c+j \omega$, thus is obtained the pole-zero diagram which gives a good overview and it allows much easier analysis for studying the dynamic behavior of each individual system.

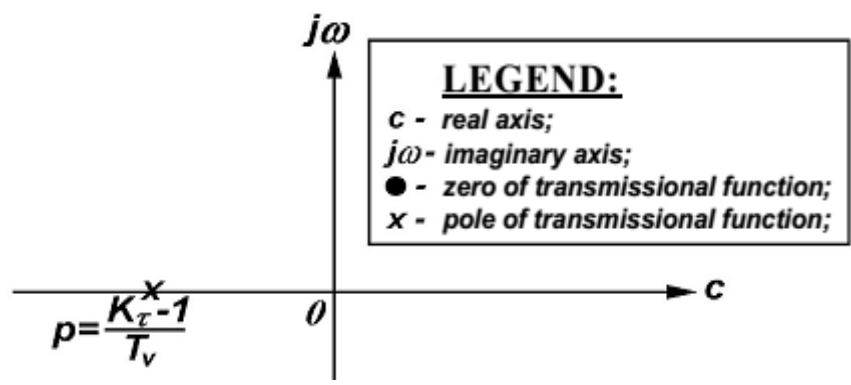

Figure 7. PN-diagram of oscillatory transporting platform during the sieving of wet earth mass.

\section{4- Frequency Response, Amplitude-phase-frequency Characteristic and Polar Diagram of Oscillatory Transporting Platform during the Sieving of Wet Earth Mass}

If from domain of a complex variable $p=c+j \omega$ we move into the domain of complex frequency $p=j \omega$ (by putting the $c=0$ ), based on Equations 5 and 7 we come to features frequencies $G(j \omega)$ which thus represents transmissional function of the observed system on the harmonic (sinusoidal) excitation:

$G(j \omega)=\frac{K_{P}}{T_{V} \cdot j \omega+1-K_{\tau}} e^{\left(\frac{1-K_{\tau}}{T_{V}}\right) \tau}$

The corresponding absolute value of frequency characteristic shown in Equation 9 represents amplitudefrequency characteristic, i.e. amplification factor of the system E-P-WP.

$|G(j \omega)|=M(\omega)=\frac{K_{P} \cdot e^{\left(\frac{1-K \tau}{T_{V}}\right) \tau}}{\sqrt{\left(1-K_{\tau}\right)^{2}+T_{V}^{2} \cdot \omega^{2}}}$

Phase-frequency characteristic shown in Equation 10 also be determined based on the frequency characteristics $G(j \omega)$, as the corresponding ratio of its imaginary and real part and the same represents delay characteristic of the output harmonic signal with regard to the harmonic input signal of the system E-P-WP.

$\operatorname{tg} \varphi=\frac{I_{m}[G(j \omega)]}{R_{e}[G(j \omega)]}=-\frac{T_{V} \cdot \omega}{1-K_{\tau}} \Rightarrow \varphi=\operatorname{arctg}\left(-\frac{T_{V} \cdot \omega}{1-K_{\tau}}\right)$

Results of complete analysis of the E-P-WP system using the frequency response method can be graphically displayed using polar diagram of the frequency characteristic, which is in literature also known under the name 
amplitude-phase-frequency characteristic of the system (Figure 8). For easier and simpler construction of this diagram for characteristic excitation frequency $\omega$ listed in the Table 1, the following assumptions have been introduced [3]:

$t_{o p}=3 T_{V}$ - response time of the platform (including time of delay $t$ );

$\tau=T_{V}$ - delay time is equal to the temporal (inertial) constant of the system;

$K_{\tau}=0.5$ - delay coefficient sifted earth relative to delivered earth (the same in reality is in a range $\left.K_{\tau}=[0 \ldots 1]\right) ;$

$e^{\left(\frac{1-K_{\tau}}{T_{V}}\right) \tau}=e^{1-K_{\tau}}=e^{1-0.5} \approx 1.65$ - operator delay of the system $E-P-W P$

Table 1. Amplitude-phase-frequency characteristics of the systems E-P-WP for different values of the excitation frequency $\omega$.

\begin{tabular}{ccccccc}
\hline Characteristic point & $\boldsymbol{\omega}$ & $\boldsymbol{R}_{e}[\boldsymbol{G}(j \omega)]$ & $\boldsymbol{I}_{\boldsymbol{m}}[\boldsymbol{G}(j \omega)]$ & $|\boldsymbol{G}(j \omega)|=\boldsymbol{M}(\boldsymbol{\omega})$ & $\boldsymbol{t} \boldsymbol{g} \boldsymbol{\varphi}=\frac{\boldsymbol{I}_{\boldsymbol{m}}[\boldsymbol{G}(\boldsymbol{j} \boldsymbol{\omega})]}{\boldsymbol{R}_{\boldsymbol{e}}[\boldsymbol{G}(\boldsymbol{j} \boldsymbol{\omega})]}$ & $\boldsymbol{0}$ \\
\hline 1 & 0 & $3.3 K_{P}$ & 0 & $3.3 K_{P}$ & 0 & $0^{\circ}$ \\
2 & $\frac{1}{4 T_{v}}$ & $2.64 K_{P}$ & $-1.32 K_{P}$ & $2.95 K_{P}$ & -0.5 & $-26.56^{\circ}$ \\
3 & $\frac{1}{2 T_{v}}$ & $1.65 K_{P}$ & $-1.65 K_{P}$ & $2.33 K_{P}$ & -1 & $-45^{\circ}$ \\
4 & $\frac{1}{T_{v}}$ & $0.66 K_{P}$ & $-1.32 K_{P}$ & $1.48 K_{P}$ & -2 & $-63.43^{\circ}$ \\
5 & $\propto$ & 0 & 0 & 0 & --- & $-90^{\circ}$ \\
\hline
\end{tabular}

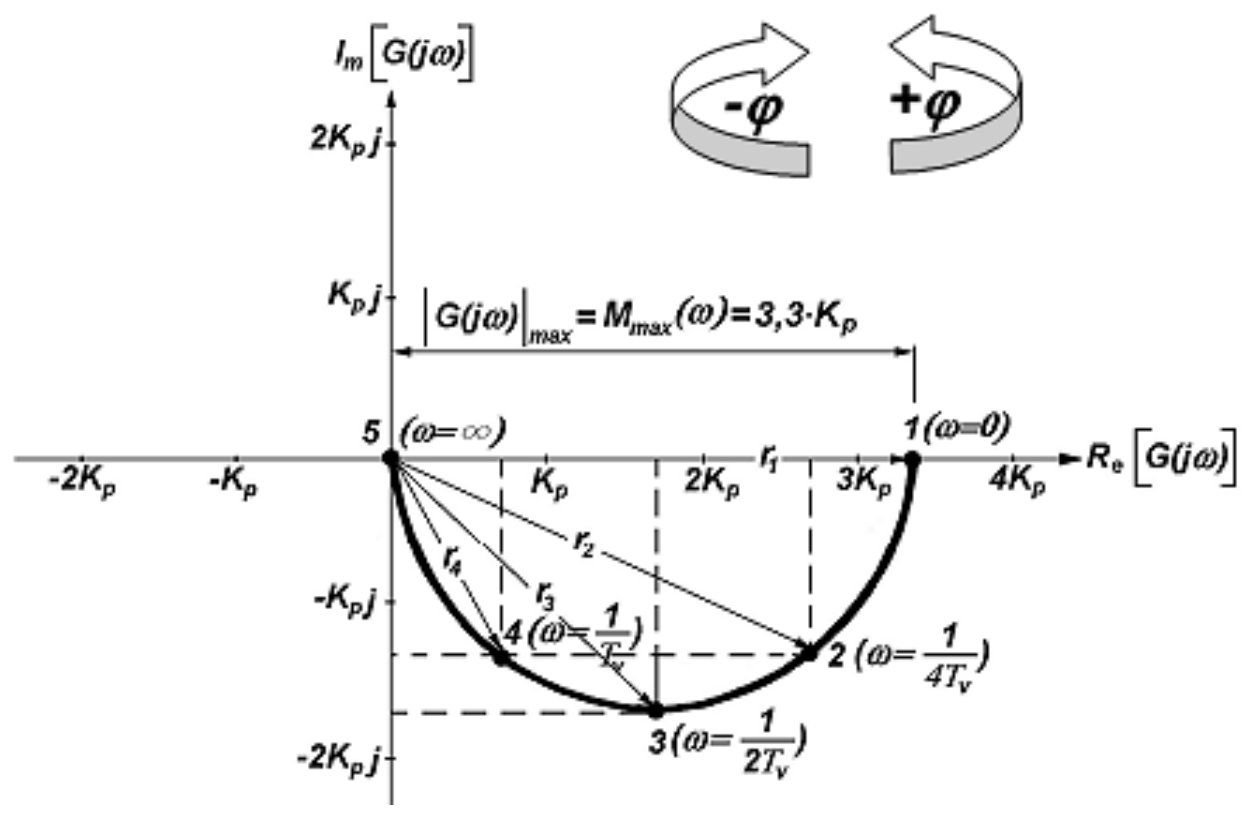

Figure 8. Polar diagram of the frequency characteristic $G(j \omega)$ of oscillatory transporting platform during the sieving of wet earth mass.

\section{5- Stability and Manageableness of Oscillatory Transporting Platform during the Sieving of Wet Earth Mass}

Under the general stability of the mechanical system is considered to be its ability to, after the cessation of any disorder, reoccupy its reference, i.e. desired position (definition according to Lyapunov). This means that: if the platform kicked out of its stable-reference position by means of any outside or any internal disorder and if the same upon the cessation of these disorders quickly "returns" to the reference position, its work is THE STABLE [2]. Otherwise, if the difference between the parameters $Q_{1}$ and $Q_{2}$ becomes bigger and bigger and after cessation of disturbance, then the work of platform is UNSTABLE, because of large deviation from the desired i.e. expected differences between delevered and sifted quantity of wet land mass.

In addition to the previous general definition of stability, it is possible to define the stability of control of platform's operation (so-called "manageableness of platform"), which is the ability of the platform to "hold" the desired operating parameters during a disturbance [15]. This practically means the following: platform under the influence of disturbances deviations from the reference values and therefore the amount of non-sieved wet earth 
mass $\left(Q^{*}=Q_{1}-Q_{2}\right)$ takes some current value $Q_{t} *$ which deviates from the reference value $Q^{*}$. After cessation of the effects of the disorder, two characteristic cases are possible:

- I case: If the values of $Q_{t}{ }^{*}$ are reduced oscillatory or asymptotically and approach the reference value $Q^{*}$, platform can be managed during disturbances, for such platform it is said to have a STABLE MANAGEABLENESS.

- II case: If the values of $Q_{t}^{*}$ are increased oscillatory or asymptotically and they are increasingly moving away from the reference value $Q^{*}$, platform can not be managed during disturbances, for such platform it is said to have an UNSTABLE MANAGEABLENESS.

Since in our case the same operating parameter (the amount of non-sieved wet earth mass $Q^{*}$ ) affects both, the general stability and the manageablenesst of the platform, for practical reasons, these two related terms will be unified into a common term - STABILITY OF THE PLATFORM and therefore, the same will be used further in consideration of the aforementioned problems.

In order to study the stability of technical systems, from all generally accepted test functions it is most appropriate Dirac's delta $\delta(t) f u n c t i o n$ ie. unit impulse function. When $\delta(t)$ function is used as a disturbance input function, the studied system will always be stable if output size of the system, so-called impulse response of the system $g(t)$ tends to zero when time tends to infinity $(t \rightarrow \infty)$. In this case, impulse response of the system corresponds to the transmissional function of the system $G(p)$, so the study of the stability of the system can only be reduced to the study of its transmissional function.

In general, the transfer function can also be displayed as a rational function in the form of a fraction $G(p)=M(p) / N(p)$, where $M(p)$ and $N(p)$ are polynomials per " $p$ " of the numerator or the denominator, respectively. When in the complex plane the poles of the transmissional function are marked with: $p_{1}, p_{2}, \ldots, p_{n}$, then the transmissional function can be written as a sum of several partial fractions:

$G(p)=\frac{M(p)}{N(p)}=\frac{A_{1}}{p-p_{1}}+\frac{A_{2}}{p-p_{2}}+\ldots+\frac{A_{n}}{p-p_{n}}$

If the inverse Laplace transformation is applied to Equation 12, a response to the disturbance $\delta(t)$ test function is obtained in the domain of real time variable (" $t$ "-domain):

$y(t)=A_{1} \cdot e^{p_{1} t}+A_{2} \cdot e^{p_{2} t}+\ldots+A_{n} \cdot e^{p_{n} t}$

$A_{1}, A_{2}, \ldots, A_{n}$ - constants determined on the basis of initial conditions of motion by the method of identity and which do not affect the stability of the system.

Poles of transmissional function $G(p): p_{1}, p_{2}, \ldots, p_{n}$ are generally complex numbers, so the stability of the studied system can be concluded only on the basis of the sign (positive / negative) of the real parts of the mentioned poles. The following is true:

$\rightarrow$ If in Equation 13 the real parts of the poles $p_{1}, p_{2}, \ldots, p_{n}$ are negative, with increasing time $t$ decreases the value of individual partial members in the equation, which means that the value of the response function $y(t)$ decreases and one in this case tends to zero $[y(t) \rightarrow 0$, if $t \rightarrow \propto]$.

$\rightarrow$ If in Equation 13 the real parts of the poles $p_{1}, p_{2}, \ldots, p_{n}$ are positive, with increasing time $t$ increased the value of individual partial members in the equation, and then the value of the response function $y(t)$ is increased and in this case it moves away from zero and tends to infinity [y(t) $\rightarrow \propto$, ако $t \rightarrow \propto]$.

Based on the previous two paragraphs, it is possible to present an important conclusion that represents the general theory of the stability of mechanical systems:

"Movement of any mechanical system will be stable only when the real parts of the poles of transmissional function $G(p): p_{1}, p_{2}, \ldots, p_{n}$ less than zero (negative)".

In practice this means that the determination of the stability of mechanical system can be reduced only to determining the position of the poles of the transmissional function $G(p)$ in the plane of complex variable $p=c+j \omega$.

All possible variants of the positions of the poles of the transmissional function $G(p)$ are shown in Figure 9, while all possible responses with the characteristic behavior of the system are given in Table 2 [13]. Graphic interpretations of Table 2, i.e. its corresponding characteristic system responses, are shown in Figure 10. 


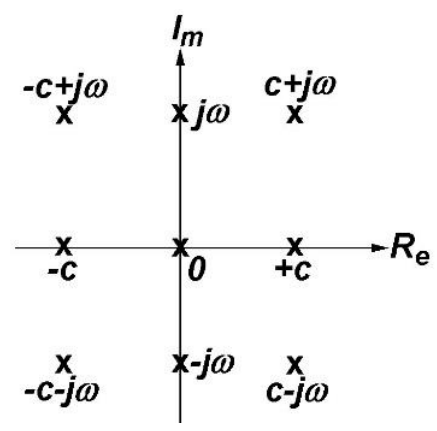

Figure 9. Possible position of the poles of transmissional function $G(p)$ in the plane of complex variable $p=c+j \omega$.

Table 2. Possible responses with characteristic behavior of the system.

\begin{tabular}{|c|c|c|c|}
\hline Ordinal number & Pole & Response & Characteristic of behavior and system state \\
\hline 1. & $-c$ & $A \cdot e^{-c t}$ & $\begin{array}{l}\text { Damped exponential function; } \\
\text { System STABLE }\end{array}$ \\
\hline 2. & 0 & $A$ & $\begin{array}{l}\text { Constant; } \\
\text { System neutral - STATIC STABLE }\end{array}$ \\
\hline 3. & $+c$ & $A \cdot e^{c t}$ & $\begin{array}{l}\text { Increasing exponential function; } \\
\text { System UNSTABLE }\end{array}$ \\
\hline 4. & $-c \pm j \omega$ & $A \cdot e^{-c t} \sin (\omega t+\varphi)$ & $\begin{array}{l}\text { Exponential damped sinusoid; } \\
\text { System STABLE }\end{array}$ \\
\hline 5. & $\pm j \omega$ & $A \sin (\omega t+\varphi)$ & $\begin{array}{l}\text { Sinusoid constant amplitude; } \\
\text { System neutral - CONDITIONAL STABLE }\end{array}$ \\
\hline 6. & $c \pm j \omega$ & $A \cdot e^{c t} \sin (\omega t+\varphi)$ & $\begin{array}{l}\text { Increasing exponential sinusoid; } \\
\text { System UNSTABLE }\end{array}$ \\
\hline
\end{tabular}
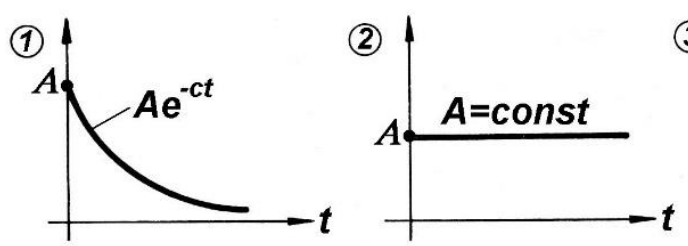

(3)
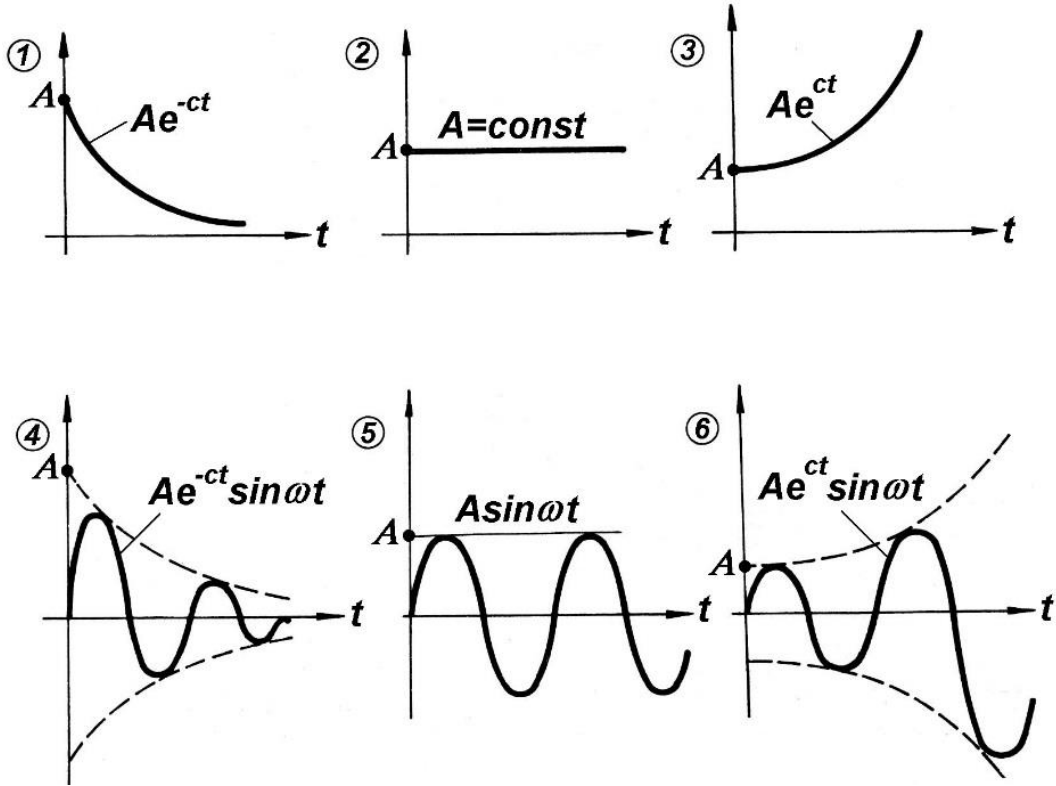

Figure 10. Typical system responses for cases listed in Table 2.

\section{6- Reserve of Stability of Oscillatory Transporting Platform during the Sieving of Wet Earth Mass}

In this case, first we have to determine the value of the maximum amplification of the system $M_{\max }(\omega)$ for the case of resonance, when the external excitation frequency is equal to its own frequency of the system [16]. For this purpose, in Equation 9 it is necessary to introduce a shift $\omega=\omega_{0}$, after which it was obtained [17]:

$|G(j \omega)|_{\max }=M_{\max }(\omega)=\frac{K_{P} \cdot e^{\left(\frac{1-K_{\tau}}{T_{V}}\right) \tau}}{\sqrt{\left(1-K_{\tau}\right)^{2}+T_{V}^{2} \cdot \omega_{0}^{2}}}$

By definition, relative stability, i.e. reserve stability of the system E-P-WP is inversely proportional to the size $M_{\max }(\omega)$ and the same is: 


$$
R_{S}=\frac{1}{M_{\text {max }}(\omega)}=\frac{\sqrt{\left(1-K_{\tau}\right)^{2}+T_{V}^{2} \cdot \omega_{0}^{2}}}{K_{P} \cdot e^{\left(\frac{1-K_{\tau}}{T_{V}}\right) \tau}}
$$

If a greater reserve stability of platform $R_{S}$, insofar there is less possibility that during resonance and at frequencies that are close to the resonant $\left(\omega \approx \omega_{0}\right)$ that may occur to disruption of the process of separation and sieving of wet earth mass. In some extreme cases [18, 19], it could happen that platform productivity falls below a minimum allowable techno-economic criteria, and even to the fact that the work of the platform is fully stopped.

\section{7- Conclusion}

The paper presents the results of testing the stability and manageableness of the oscillatory transporting platform during the sieving of wet earth mass. All relevant results were obtained on the basis of a dynamic analysis of the mentioned processes, based on the mathematical model given by the Equation 3. On the basis of transmissional function $G(p)$ it has been found that the studied oscillatory transport platform represents a specific combination which in the theory of modeling is known under the name aperiodic element of the first order with time delay. Corresponding "PNdiagram" of this element is shown in Figure 7.

If all the above considerations are applied to our studied system E-P-WP, it can be concluded the following:

- Based on the Equations 5 and 6 and bearing in mind the fact that it is always $K_{\tau}<1$ (because the case $K_{\tau}=1$ is possible mathematically but not physically), it can be concluded that the real part of the pole " $p$ " of transmissional function $G(p)$ is always less than zero (it's always negative), so the system E-P-WP is a STABLE mechanical system.

- Based on Figures 7 and 9, the pole of transmissional function $G(p)$ can be generally written in the form $p=\frac{K_{\tau}-1}{T_{V}}=-c$.

- Based on the Table 2 and Figure 10 it is obvious that this is a case listed under number 1, for which the imaginary value of the pole of the transmission function $G(p)$ is zero $(j \omega=0)$ and for which the studied system E-P-WP is STABLE.

- Polar diagram (Figure 8) shows the dependence of the amount of sieved wet earth mass $Q_{2}$ (output size) from different frequencies of oscillation of external disturbing forces (input size). After analyzing the polar diagram, it is possible to validate the following facts:

* For $\omega=0$ and $\varphi=0^{\circ} \Rightarrow$ System amplification is the highest and its absolute value is: $|G(j \omega)|=M(\omega)=3,3 K_{P}$;

* With further increase in the value of $\omega$ amplification of the system has a tendency of continuous reduction;

$*$ For $\omega=\propto$ and $\varphi=-\pi \mathrm{rad}=-180^{\circ} \Rightarrow$ amplification of the system disappears (it takes the value zero).

- On the basis of the Equation 15 reserve stability of the system E-P-WP is greater if are larger the inertia of platform (expressed through temporal constant $T_{V}$ ) and eigenfrequencies of oscillation of the platform $\left(\omega_{0}\right)$, while simultaneously, amplification factor of the platform $\left(K_{P}\right)$ and the delay time of the output signal relative to the input signal $(\tau)$ should have the smallest possible value.

- The results and conclusions obtained by the authors of this paper could be the starting point i.e. the base for any further similar or new research into the above mentioned issues:

* Our experimental platform has one sieve (mono-grid platform) and it is known that in practice dual-grid platforms have been implemented. Studying the transportation and separation of wet earth mass on a dualgrid platforms would produce very interesting results, because the working performance (productivity) of dual-grid platforms is significantly higher than mono-grid platforms. This means that a mathematical model which describing the operation of dual-grid platforms should consist of a system of two differential equations, where one equation relates to the first sieve and the second equation relates to the second sieve.

* Our experimental platform is characterized by the harmonic movement of the working body (coulter) for undermining of the earth, in which the speeds and accelerations change according to sine (cosine) law. Some authors claim that in the case of non-harmonious movement of the working body, the rate of undermining of the wet mass can be increased up to $15 \%$. Therefore, an original research is proposed with a platform whose working body would perform non-harmonic movement, why, in the opinion of the authors of this paper, is the most suitable platform to have a hydraulic drive instead of a mechanical one. 
* It would be interesting to have a new theoretical modeling in which a nonlinear mathematical model would be applied instead of a linear one. Nonlinear differential equations impose some completely different criteria for dynamic analysis and stability testing of the studied system. However, the results thus obtained would be much more relevant, since most real phenomena and processes in technical systems are closer to nonlinear than linear behavior.

\section{8- Conflict of Interest}

The author declares that there is no conflict of interests regarding the publication of this manuscript. In addition, the ethical issues, including plagiarism, informed consent, misconduct, data fabrication and/or falsification, double publication and/or submission, and redundancies have been completely observed by the authors.

\section{9- References}

[1] G. Mihajlović. "Dynamical Modelling of the Process of Separation and Sieving of Wet Land Mass by Oscillatory Transporting Platform." IMK-14- Research and Development in Heavy Machinery, 20(1) (2014).

[2] G. Mihajlović, M. Gašić, and M. Savković. "Research on Parameters Influencing Performance of Vibrating Platforms during Transporting the Dry Earth Mass." Tehnicki Vjesnik - Technical Gazette 24, no. Supplement 1 (May 2017): 127-135. doi:10.17559/tv-20150402111852.

[3] G. Mihajlović. "Influential parameters on the working ability of oscillatory transporting platforms for sieving moist materials." Faculty of Mechanical and Civil Engineering, Kraljevo, University of Kragujevac, Doctoral Thesis (2017).

[4] G. Mihajlović, and D. Golubović. "Breaking a solid mass by means of vibration-swinging oscillating platform," (original scientific paper). 1998 Symposium "Contemporary agriculture", Technical Faculty and Agronomy Faculty, Čačak, (4-7 November 1998), pp. 231-236 UDK.531.3.

[5] S. Timoshenko, D. Young, and W. Weaver. "Vibration Problems in Engineering, 4th edition " (1974). John Wiley and Sons, New York.

[6] Shemyakin, E.I., N.P. Benevolenskaya, and A.Ya. Tishkov. "Vibration Machines and Man." Studies in Environmental Science (1981): 348-352. doi:10.1016/s0166-1116(09)70161-x.

[7] Dašić, P. "Response surface methodology: Selected scientific-professional papers." SaTCIP Publisher Ltd. (2019): 306 (in Serbian).

[8] Dašić, P. "Comparative analysis of different regression models of the surface roughness in finishing turning of hardened steel with mixed ceramic cutting tools." Journal of Research and Development in Mechanical Industry (JRaDMI) 5, no. 2 (2013): 101-180.

[9] Yuvaraju, B.A.G., and B.K. Nanda. "Prediction of Vibration Amplitude and Surface Roughness in Boring Operation by Response Surface Methodology.” Materials Today: Proceedings 5, no. 2 (2018): 6906-6915. doi:10.1016/j.matpr.2017.11.352.

[10] Babu, GHV Prasad, BSN Murthy, K Venkatarao, and Ch Ratnam. "Multi-Response Optimization in Orthogonal Turn Milling by Analyzing Tool Vibration and Surface Roughness Using Response Surface Methodology." Proceedings of the Institution of Mechanical Engineers, Part B: Journal of Engineering Manufacture 231, no. 12 (January 15, 2016): $2084-2093$. doi: $10.1177 / 0954405415624349$.

[11] Boutaghane, Fateh, Hamdi Aouici, and Abdelaziz Mahmoud Bouchelaghem. "Classification of the Vibration Conditions on the Natural Frequency and the Maximal Displacement Using Response Surface Methodology (RSM)." The International Journal of Advanced Manufacturing Technology 103, no. 9-12 (May 29, 2019): 4495-4505. doi:10.1007/s00170-019-03867-z.

[12] G. Mihajlović. "Determination of optimal operating parameters of vibration-swinging oscillating platforms from the aspect of breaking and separating the transporting mass." Technical Faculty, Čačak, University of Kragujevac, Master Thesis (1995).

[13] D. Simić. "The basics of automatic control" (1984). Building book, Belgrade.

[14] Kim, Chang-Ju, Jeong-Seok Oh, and Chun-Hong Park. "Modelling Vibration Transmission in the Mechanical and Control System of a Precision Machine.” CIRP Annals 63, no. 1 (2014): 349-352. doi:10.1016/j.cirp.2014.03.133.

[15] Encyclopedia. "Tekniken" (1984). Focus International Book Produktion, Stockholm.

[16] D. Golubović. "The dynamics of the system / Stability of driving a car", monograph (1990). Faculty of Engineering, Čačak.

[17] G. Mihajlović, and D. Golubović. "Optimization of geometrical parameters of vibration-swinging oscillating platform." 2002 Scientific conference IRMES, Jahorina, (19-20 September 2002), pp. 595-600.

[18] Krishnamurthy, K., and M.-C. Chao. “Active Vibration Control During Deployment of Space Structures.” Journal of Sound and Vibration 152, no. 2 (January 1992): 205-218. doi:10.1016/0022-460x(92)90356-3.

[19] Krolczyk, Grzegorz M., Jolanta B. Krolczyk, Stanislaw Legutko, and Anica Hunjet. "Effect of the disc processing technology on the vibration level of the chipper during operations." Tehnički vjesnik 21, no. 2 (2014): 447-450. 